\title{
Photoplethysmographic Waveform in Hyperbaric Environment*
}

\author{
María Dolores Peláez Coca ${ }^{1,2}$, Alberto Hernando ${ }^{1,2}$, Carlos Sánchez ${ }^{1,2}$, \\ María Teresa Lozano Albalate ${ }^{1,2}$, David Izquierdo ${ }^{1,3}$ and Eduardo Gil ${ }^{2}$
}

\begin{abstract}
The objective of this work is the identification of significant variations of morphological parameters of the photoplethysmographic (PPG) signal when the subjects are exposed to an increase in atmospheric pressure. To achieve this goal, the PPG signal of 26 subjects, exposed to a hyperbaric environment whose pressure increases up to $5 \mathrm{~atm}$, has been recorded. From this record, segments of 4 minutes have been processed at $1 \mathrm{~atm}, 3 \mathrm{~atm}$ and $5 \mathrm{~atm}$, both in the descending $(D)$ and ascending $(A)$ periods of the immersion. In total, four states $(3 D, 5,3 A$ and $1 A)$ normalized to the basal state $(1 D)$ have been considered. In these segments, six morphological parameters of the PPG signal were studied. The width, the amplitude, the widths of the anacrotic and catacrotic phases, and the upward and downward slopes of each PPG pulse were extracted. The results showed significant increases in the three parameters related to the pulse width. This increase is significant in the four states analysed for the anacrotic phase width. Furthermore, a significant decrease in the amplitude and in both slopes (in the states $1 A$ ) was observed. These results show that the PPG width responds rapidly to the increase in pressure, indicating an activation of the sympathetic system, while amplitude and pulse slopes are decreased when the subjects are exposed to the hyperbaric environment for a considerable period of time.
\end{abstract}

\section{INTRODUCTION}

The health safety of divers is essential. Our body has to adapt to the conditions of the environment while maintaining the internal equilibrium needed for survival. An immersion in water represents a considerable physiological challenge of the regulatory system to maintain homeostasis [1]. The capacity to respond to the challenge of immersion in water highly depends on the physiological systems that control blood pressure and heart rate in short term. In particular, the autonomic nervous system (ANS) presents a rapid response to changes in blood pressure induced by immersion.

Poiseuille's law states that the increase in the cardiac output produced by immersion will increase the blood pressure if the radius of the blood vessels remains constant. This increment will trigger the homeostatic mechanisms to mitigate this effect, with the barorreflex system being

\footnotetext{
*This work was not supported by any organization

${ }^{1}$ M.D. Peláez, A. Hernando, C. Sánchez and M. Lozano, are with Centro Universitario de Defensa (CUD), Zaragoza, Spain. (mdpelaez@unizar.es, ahersanz@unizar.es, cstapia@unizar.es, maytelo@unizar.es, d.izquierdo@unizar.es)

${ }^{2}$ M.D. Peláez, A. Hernando, C. Sánchez, M. Lozano and E. Gil are with BSICoS group, Aragón Institute of Engineering Research (I3A), IIS Aragón, University of Zaragoza, Zaragoza, Spain and A. Hernando and E. Gil is with Centro de Investigación Biomédica en Red Bioingeniería, Biomateriales y Nanomedicina (CIBER-BBN), Madrid, Spain. (edugilh@unizar.es)

${ }^{3} \mathrm{D}$. Izquierdo are with GTF group, Aragón Institute of Engineering Research (I3A), University of Zaragoza, Zaragoza, Spain
}

responsible for the initial response. During immersion, in resting conditions, the increments in both the central blood volume and the pressure result in a decrease in the heart rate [1]. Due to the increase of the cardiac preload and the systolic volume, a decrease in the heart rate allows to maintain an adequate cardiac output at the same time that the increase in blood pressure induced by hydrostatic pressure is minimized. The effects of immersion on the cardiovascular system are clearly visible in the cardiac output, calculated as the product of systolic volume and heart rate. An increase in the central blood volume entails an increase in the venous return and thus in both the systolic volume [2] and the cardiac output [3].

It has also been shown that, in addition to a reduction in their heart rate highly dependent on the time spent in the hyperbaric environment, the exposure of a subject to a hyperbaric environment produces a reduction in the values of the sympathetic markers. A variation of the high-frequency (HF) power of the heart rate variability, with a positive correlation with the variation of atmospheric pressure has been shown as well [4]. These subjects also experience a higher density of breathing gases, increased cardiac output, and, in the case of immersion in water, changes in blood distribution, increased resistance to movement, changes in temperature due to cold water, etc. All these changes have an impact on the PPG morphology.

The PPG signal, recorded on the finger, reflects the wavy movement of the blood in the blood vessels. This movement is affected by heartbeats, hemodynamics and changes in the properties of the finger arterioles. Apart from the hyperbaric effects, these effects can be observed as distortions in the PPG wave profiles, and some of them have been related to physiological changes of the subjects [5], [6]. The objective of this work is to prove that changes in either atmospheric or hydrostatic pressure cause a physiological response (alterations in blood distribution, increase in cardiac output, decrease in heart rate, changes in the power of the HF band, etc.) that may introduce distortions in the waveform of the PPG signal. Therefore, in this work, an analysis of the PPG signal morphology was proposed in order to identify the most relevant features showing significant changes with atmospheric pressure variations.

\section{MATERIALS}

A total of 26 subjects (22 males and 4 females), with a mean age of $28.7 \pm 6.4$ years and a strong component of military personnel (21 out of 26) were recorded in the study. After receiving approval for the study from the Comite de 
ética de la investigación con medicamentos de la inspección general de sanidad de la Defensa (Ethics Committee of Research with Medicines), all subjects were recorded inside the hyperbaric chamber of the Hospital General de la Defensa de Zaragoza. The protocol inside the chamber had a duration of about two hours. Five different stages, in five control stops, involving $5 \mathrm{~min}$ at $1 \mathrm{~atm}$ (sea level), 3 atm (equivalent to $20 \mathrm{~m} \mathrm{depth}$ ) and $5 \mathrm{~atm}$ (equivalent to $40 \mathrm{~m} \mathrm{depth}$ ), and subsequently returning to $3 \mathrm{~atm}$ and 1 atm were performed. Most of the time was spent in the decompression stops between $3 \mathrm{~atm}$ and $1 \mathrm{~atm}$, as recommended in standard decompression tables. In 21 subjects, this decompression time was $44 \mathrm{~min}$. In the rest of the cases, this time was slightly shorter, but always longer than 30 min. Subjects stayed relaxed and sitting comfortably, and the chamber was correctly ventilated throughout the protocol in an attempt to avoid major changes in temperature and humidity. Subjects remained in silence and without perform movements during control stops. Therefore, a total of five stages, referred to as $1 D$ (basal stage), $3 D, 5,3 A$ and $1 A$ (the number reflects the pressure in atm; the letter D or A refers to descent or ascent period) were studied. Recordings were performed using a Nautilus device, which digitalizes the finger PPG signal at a sampling frequency (fs) of $1000 \mathrm{~Hz}$ and the chamber atmospheric pressure with a fs of $250 \mathrm{~Hz}$.

\section{METHODS}

\section{A. Morphological parameters of PPG signal}

First, PPG pulses were automatically detected using an algorithm based on a low-pass differentiator filter [7]. Then, the apex $\left(n_{\mathrm{Ai}}\right)$, the basal $\left(n_{\mathrm{Bi}}\right)$, the onset $\left(n_{O i}\right)$ and end $\left(n_{E i}\right)$ points of the $i$-th PPG pulse (Fig. 1) were identified as in [8]. In this work, six morphological parameters of the PPG signal are considered. The amplitude $(P A)$ and the width $(P W)$ are estimated based on the following equations.

$$
\begin{gathered}
P A(i)=x_{P P G}\left(n_{A i}\right)-x_{P P G}\left(n_{B i}\right) \\
P W(i)=\frac{1}{f_{s}}\left(n_{E i}-n_{O i}\right)
\end{gathered}
$$

The next two parameters also define the PPG wave width. First segment corresponds to the anacrotic phase of the pulses $\left(P W_{u}\right)$, related to the upward segment of the pulses produced during the systole [6], the second segment corresponds to the catacrotic phase $\left(P W_{d}\right)$, related to the downward segment of the pulses produced during diastole and wave reflections from the periphery [6]. The maximum amplitude of the pulse $n_{A i}$ is the point of separation between them. The parameters are estimated as:

$$
P W_{u}(i)=\frac{1}{f_{s}}\left(n_{A i}-n_{O i}\right) \quad P W_{d}(i)=\frac{1}{f_{s}}\left(n_{E i}-n_{A i}\right)
$$

The last two morphological parameters are defined as the upward and downward slopes of each PPG pulse, $P S_{u}$ and $P S_{d}$, respectively. These two parameters are calculated as the ratios between the amplitude and the width of each upward and downward segment, respectively:

$$
\begin{aligned}
& P S_{u}(i)=f_{s} \frac{x_{P P G}\left(n_{A i}\right)-x_{P P G}\left(n_{O i}\right)}{n_{A i}-n_{O i}} \\
& P S_{d}(i)=f_{s} \frac{x_{P P G}\left(n_{A i}\right)-x_{P P G}\left(n_{E i}\right)}{n_{E i}-n_{A i}}
\end{aligned}
$$

An outlier identification was carried out on all morphological parameters based on [9], where every parameter outside the range [median $\pm G \cdot s t d]$ of the $N_{g}$ previous pulses is removed. In this study $G=5$ and $N_{g}=50$.

In summary, based on the significant points of each PPG pulse (Fig. 1), the following morphological parameters are defined as the mean of the four minutes selected for each state $(1 D, 3 D, 5,3 A$ and $1 A)$ :

- $\overline{P W}$ : Mean width of the pulses (in seconds);

- $\overline{P A}$ : Mean amplitude of the pulses (arbitrary units);

- $\overline{P W_{u}}$ and $\overline{P W_{d}}$ : Mean width of the anacrotic and catacrotic phases of the pulses (in seconds);

- $\overline{P S_{u}}$ and $\overline{P S_{d}}$ : Mean upward and downward slopes of the PPG pulses (arbitrary units).

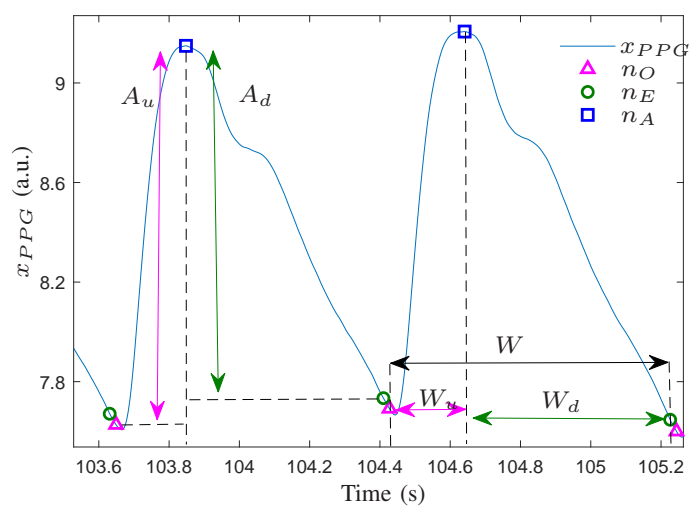

Fig. 1. Location of the start $n_{O i}$, the end $n_{E i}$ and the apex $n_{A i}$ points, used to calculate the morphological parameters of the PPG signal. (a.u.: arbitrary units)

Every parameter was normalized to the sum of the reference state $(1 D)$ :

$$
\mathcal{R}\left(\mathcal{Y}_{S}^{k}\right)=\mathcal{Y}_{S}^{k} /\left(\mathcal{Y}_{S}^{k}+\mathcal{Y}_{1 D}^{k}\right)
$$

where $\mathcal{Y}$ is the mean of each one of the six parameters $(\overline{P W}$, $\overline{P A}, \overline{P W_{u}}, \overline{P W_{d}}, \overline{P S_{u}}$ and $\left.\overline{P S_{d}}\right)$ in each segment $S(1 D, 3 D$, $5,3 A$ and $1 A$ ) for each subject $k$, and $k=1 \ldots N$, where $N$ is the number of subjects. Parameters have been normalized to minimize the effects of the intersubject variance.

\section{B. Statistical analysis}

A statistical analysis of the six parameters obtained from the PPG signals was implemented in order to identify significant differences with respect to the reference state $(1 D)$.

The Shapiro-Wilk test is applied to distinguish whether the parameters have a normal distribution. The t-Student test is applied if the distribution is normal, otherwise, the Wilcoxon paired test is the one applied. A $p$-value $<\alpha$ defines significance, where the significance level $\alpha$ can be $0.05,0.01$ 
or 0.001 . These tests are applied to each one of the six normalized parameters extracted from the PPG signals.

\section{RESULTS}

The results of Fig. 2 show a significant increase in the PPG width, when subjects are exposed to an increase in atmospheric pressure. This increase, for $\overline{P W}$ and $\overline{P W_{u}}$, is maintained throughout the entire immersion and it can be observed that it is also significant in all states, except in the maximum pressure (5 atm) for $\overline{P W}$. For $\overline{P W_{d}}$, this increase is significant only in the last two stages ( $3 A$ and $1 A$ ). The $\overline{P A}$ remains without significant changes during the states of increased pressure. However, a significant decrease in amplitude was observed in the last stage, when the subjects returned to the baseline state of $1 \mathrm{~atm}$. On the other hand, there is a significant decrease in both slopes of the PPG pulse when the time the subjects are exposed to a hyperbaric environment increases.

\section{DISCUSSION}

The purpose of this study is the identification of morphological parameters of the PPG signal, which vary significantly when subjects are exposed to a variable hyperbaric environment. The width and amplitude of PPG pulse were selected as morphological parameters of interest, together with other four parameters derived from these two. These parameters were selected since it is known that the reduction of the PPG amplitude can be attributable either to a loss of central blood pressure or to constriction of the arterioles perfusing the skin, that will be influenced by sympathetic activity [6]. However, other works suggest that the pulse width correlates with the systemic vascular resistance better than the amplitude [10]. The other four selected parameters, $P W_{u}, P W_{d}, P S_{u}$ and $P S_{d}$, provide information on which segment of the PPG pulse the change occurs, anacrotic or catacrotic phase. Among the parameters studied, the amplitude and width of the PPG pulses showed a strong correlation with the activation of the sympathetic system [6], [10], although a weaker relationship was found with the pulse slopes [10].

In this study, results show a significant increase in the $\overline{P W_{u}}$, with respect to the baseline, in the four analysed states $(3 D, 5,3 A$ and $1 A)$. This increase is also observed in the width of the PPG pulse, and in some stages of $\overline{P W_{d}}$.

A limitation of this work is the difficulty to estimate $n_{E i}$ in a robust way. This is due to the fact that the downward slope of the pulse is irregular, and the algorithm can confuse a change of slope with the end of the pulse. To minimize this error, the pulse wave is smoothed before estimation. This problem does not arise in the estimation of $n_{O i}$ and $n_{A i}$, since the upward slope of the pulse is more abrupt and regular. Therefore, it is considered that the estimation of $\overline{P W_{u}}$ is robust, and that the differences with the results obtained with $\overline{P W}$ and $\overline{P W_{d}}$ can be due to these limitations in the estimation of $n_{E i}$.

Another work showed that there is an increase in the width of the PPG signal as the systemic vascular resistance increased, due to the predominantly adrenergic innervation of the finger, and therefore activated by the sympathetic system [10], [11]. On this basis, the increase of $\overline{P W_{u}}, \overline{P W_{d}}$ and $\overline{P W}$ observed in our results indicates an activation of the subjects' sympathetic nervous system when the subjects are exposed to a hyperbaric environment. This change in PPG width is maintained when the subjects return to the baseline state of $1 \mathrm{~atm}$, which suggests that the subjects need a recovery time after immersion. In these variables, there is not observed correlation with the pressure achieved or with the time elapsed in the hyperbaric environment.

Regarding the $\overline{P A}$, only a significant decrease in the state $1 A$ is seen, with respect to the baseline state, caused by an activation of the sympathetic system [6]. This same effect has been observed in subjects exposed to hyperbaric oxygen treatments [12], where the subjects are exposed to partial pressures of oxygen of at least 2 atmospheres absolute. In our study, subjects must spend a considerable period of time at pressures not exceeding $3 \mathrm{~atm}$, during the decompression stops. It is in this period of time, where an decrease of the $\overline{P A}$ is observed.

In the parameters $\overline{P S_{u}}$ and $\overline{P S_{d}}$, a significant decrease is observed for the stage $1 A$. This decrease in the slopes is due to the increase in the width of each PPG segment and the decrease in the pulse amplitude. $\overline{P A}, \overline{P S_{u}}$ and $\overline{P S_{d}}$ are parameters whose changes appear with the increase of time spent in a hyperbaric environment. No correlation is observed with the pressure in these parameters, and when the subjects return to 1 atm ( $1 D$ state) the decrease of the three parameters is maintained.

Several authors consider that the power of the Pulse Rate Variability (PRV) in the low-frequency (LF) band normalized with respect to the powers in $\mathrm{LF}$ and $\mathrm{HF}$ and the ratio between LF and HF power bands, $\left(P_{L F n}\right.$ and $R_{L F / H F}$ respectively), are a representation of the sympathetic response of the ANS [4], [13], [14]. However, an analysis of these parameters, in the same population object of this study, did not show an increase of these parameters neither with the increase of atmospheric pressure, nor with the time that the subjects spend in the hyperbaric environment [4]. Even the results showed a significant decrease of the $P_{L F n}$ in the states 5 and $3 A$, with respect to the baseline state [4]. The origin of this apparent discrepancy may lie in the fact that the finger circulation are innervated by sympathetic adrenergic nerves, which release norepinephrine as a neurotransmitter. The liberation of norepinephrine provides basal constrictor activity to the skin circulation, particularly in the extremities, and a paradoxical decrease in heart rate, due to a baroreceptor reflex, in response to the increase in arterial pressure [11]. This decrease in heart rate was also observed in the previous study [4].

\section{CONCLUSIONS}

In this work, the PPG signal of 26 subjects exposed to a variable hyperbaric environment was recorded. An analysis of PPG waveform was performed. The results show an increase in the pulse width of the PPG and a decrease in 


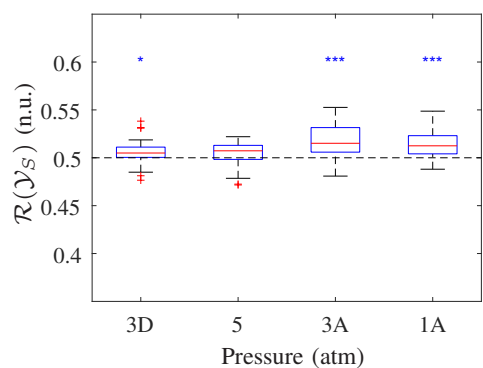

(a) $\overline{P W}$ parameter

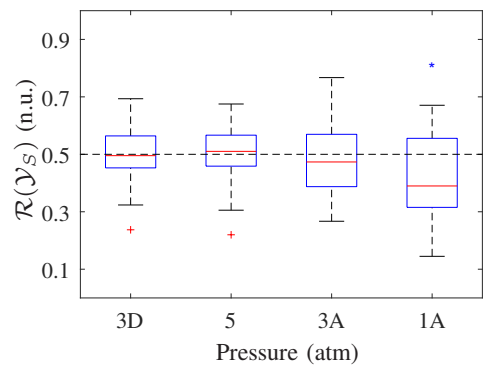

(d) $\overline{P A}$ parameter

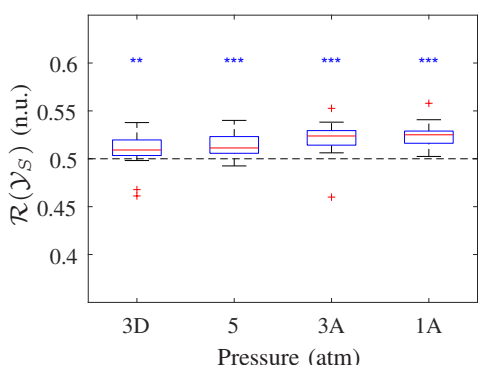

(b) $\overline{P W_{u}}$ parameter

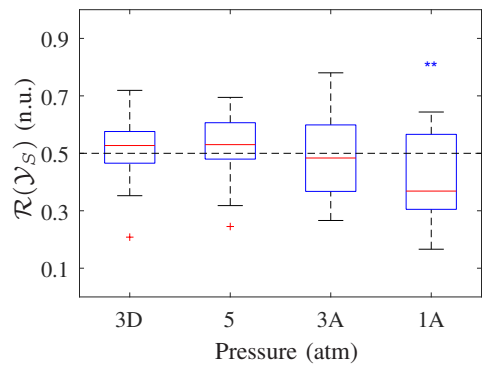

(e) $\overline{P S_{u}}$ parameter

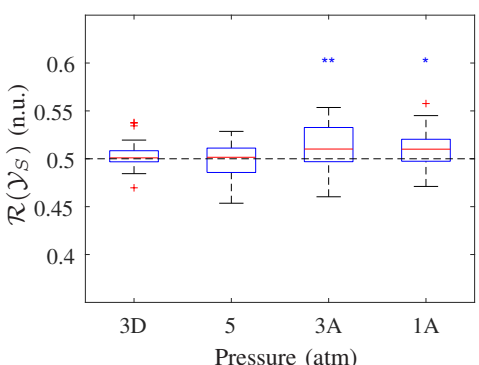

(c) $\overline{P W_{d}}$ parameter

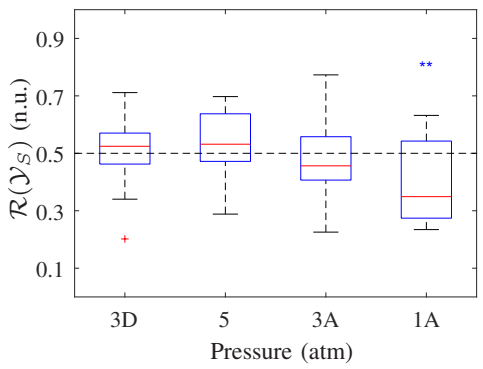

(f) $\overline{P S_{d}}$ parameter

Fig. 2. Boxplots of the $\mathcal{R}\left(\mathcal{Y}_{S}\right)$ of morphological parameters in each state analysed $(3 D, 5,3 A$ and $1 A)$ respect the basal state $(1 D)$. The significance level $\alpha$ is indicated with $*$ for $\alpha=0.05, * *$ for $\alpha=0.01$ and $* * *$ for $\alpha=0.001$. (n.u.:normalized units)

the pulse amplitude and slopes. These changes are due to an activation of the sympathetic system of the subjects when they are exposed to the hyperbaric environment. The morphological parameters of the PPG have also shown sensitivity to detect an activation of the peripheral sympathetic system, that does not affect the classic parameters of the PRV, $P_{L F n}$ and $R_{L F / H F}$. As future work, the estimation of the pulse end point will be improved and a comparison with other bioparameters will be made.

\section{ACKNOWLEDGMENT}

This work has been partially financed by Ministerio de Economía, Industria y Competitividad and by funds FEDER through the project RTI2018-097723-B-I00; by Centro Universitario de la Defensa (CUD) under the projects CUD201808 and UZCUD2017-TEC-04; and by Gobierno de Aragón (Reference Group BSICoS T39-17R) cofunded by FEDER 2014-2020. This work would never have been done without the collaboration of the Hospital General de la Defensa en Zaragoza and the assistance as volunteers of the Regimiento de Pontoneros y Especialidades de Ingenieros $n^{\circ} 12$.

\section{REFERENCES}

[1] E. P. Widmaier, H. Raff, and T. Kevin, "Vander's Human Physiology," in Vander's Human Physiology. The mechanisms of body fuction, 2013, vol. 1, no. September 2013, pp. 1-806.

[2] L. M. Ueno, M. Miyachi, T. Matsui, K. Takahashi, K. Yamazaki, K. Hayashi, S. Onodera, and T. Moritani, "Effect of aging on carotid artery stiffness and baroreflex sensitivity during head-out water immersion in man," Brazilian Journal of Medical and Biological Research, vol. 38, no. 4, pp. 629-637, 2005.

[3] L. E. Farhi and D. Linnarsson, "Cardiopulmonary readjustments during graded immersion in water at 35 C," Respiration Physiology, vol. 30, no. 1-2, pp. 35-50, 1977.
[4] A. Hernando, M. Peláez, M. T. Lozano, M. Aiger, D. Izquierdo, A. Sanchez, M. I. Lopez-Jurado, J. I. Moura, J. Fidalgo, J. Lázaro, and E. Gil, "Autonomic nervous system measurement in hyperbaric environments using ECG and PPG signals," IEEE Journal of Biomedical and Health Informatics, vol. 23, no. 1, pp. 132-142, Jan 2019.

[5] M. Peláez, M. T. Lozano, A. Hernando, M. Aiger, and E. Gil, "Photoplethysmographic waveform versus heart rate variability to identify low stress states. attention test," IEEE Journal of Biomedical and Health Informatics, pp. 1-1, 2018.

[6] M. Elgendi, "On the analysis of fingertip photoplethysmogram signals," Current Cardiology Reviews, vol. 8, no. 1, pp. 14-25, 2012.

[7] J. Lázaro, E. Gil, J. M. Vergara, and P. Laguna, "Pulse rate variability analysis for discrimination of sleep-apnea-related decreases in the amplitude fluctuations of pulse photoplethysmographic signal in children," IEEE Journal of Biomedical and Health Informatics, vol. 18, no. 1, pp. 240-246, 2014.

[8] J. Lázaro, E. Gil, R. Bailón, A. Mincholé, and P. Laguna, "Deriving respiration from photoplethysmographic pulse width," Medical and Biological Engineering and Computing, vol. 51, no. 1-2, pp. 233-242, 2013.

[9] R. Bailón, L. Sörnmo, and P. Laguna, "A robust method for ECGbased estimation of the respiratory frequency during stress testing," IEEE Transactions on Biomedical Engineering, vol. 53, no. 7, pp. 1273-1285, 2006.

[10] A. A. Awad, A. S. Haddadin, H. Tantawy, T. M. Badr, R. G. Stout, D. G. Silverman, and K. H. Shelley, "The relationship between the photoplethysmographic waveform and systemic vascular resistance," Journal of Clinical Monitoring and Computing, vol. 21, no. 6, pp. 365-372, 2007.

[11] R. E. Klabunde, "Cardiovascular physiology concepts," Lippincott Williams \& Wilkins, p. 256, 2004

[12] T. Chonis and J. S. Cooper, Hyperbaric, Cardiovascular Effects, 2018. [Online]. Available: http://www.ncbi.nlm.nih.gov/pubmed/29489293

[13] A. Hernando, J. Lazaro, E. Gil, A. Arza Valdes, J. Garzon-Rey, R. Lopez-Anton, C. de la Camara, P. Laguna, J. Aguilo, and R. Bailón, "Inclusion of respiratory frequency information in heart rate variability analysis for stress assessment." IEEE Journal of Biomedical and Health Informatics, pp. 1-1, 2016.

[14] G. G. Berntson, J. T. Cacioppo, and A. Fieldstone, "Illusions, arithmetic, and the bidirectional modulation of vagal control of the heart," Biological Psychology, vol. 44, pp. 1-17, 1996. 